\title{
Meson excitation at finite chemical potential
}

\author{
A. Hajilou ${ }^{\mathrm{a}}$, M. Ali-Akbari ${ }^{\mathrm{b}}$ \\ Department of Physics, Shahid Beheshti University G.C., Evin, Tehran 19839, Iran
}

Received: 4 August 2018 / Accepted: 11 March 2019 / Published online: 20 March 2019

(C) The Author(s) 2019

\begin{abstract}
We consider a probe stable meson in the holographic quark-gluon plasma at zero temperature and chemical potential. Due to the energy injection into the plasma, the temperature and chemical potential are increased to arbitrary finite values in such a way that the plasma experiences an out-of-equilibrium process. We then observe that the meson is excited, i.e. the expectation value of Wilson loop oscillates around its static value with a specific angular frequency. By defining excitation time $t_{e x}$ as a time at which the meson falls into the final excited state, we study the effect of various parameters of theory on the excitation time and observe that for larger values of final temperature and chemical potential the excitation time increases. Furthermore, our outcomes show that the more stable mesons, meaning that the meson with lower static potential, are excited sooner.
\end{abstract}

\section{Contents}

1 Introduction . . . . . . . . . . . . . 1

2 Review on the backgrounds . . . . . . . . . . 2

2.1 RN-AdS black hole background . . . . . . . 2

2.2 RN-AdS-Vaidya background . . . . . . . . 3

3 Expectation value of Wilson loop . . . . . . . . . 3

4 Numerical results . . . . . . . . . . . . . . . . . 4

5 Conclusions ............... . . 7

Appendix A: Large chemical potential limit . . . . . . 8

Appendix B: Boundary condition . . . . . . . . . . . . 9

Appendix C: Initial condition . . . . . . . . . . . . . 10

References . . . . . . . . . . . . . . . . 10

a e-mail: a_hajilou@sbu.ac.ir

be-mail: m_aliakbari@sbu.ac.ir

\section{Introduction}

Standard methods applied to calculations in quantum chromodynamics (QCD) are often based on a perturbative expansion. This makes them of limited use if applied to questions about the non-perturbative nature of the theory at low energies and where the coupling constant is large. Strong coupling techniques are in particular needed for understanding the physics of the strongly coupled quark-gluon plasma (QGP) produced at RHIC and at the LHC [1]. One such technique is lattice gauge theory that successfully describes the low energy properties of QCD especially at $\mu=0$ [2]. Lattice gauge theory, however, is of limited use in describing time-dependent quantities and non-equilibrium evolution. Another technique is gauge-gravity duality that we apply in this paper to explain the behavior of meson in nonequilibrium plasma in the presence of non-zero chemical potential.

The anti de-Sitter/conformal field theory (AdS/CFT) correspondence or more generally gauge-gravity duality [1,3-5] is a conjectured relation between two physical theories. One of them is a strongly coupled gauge theory in $d$ dimensional space-time and the other one is a classical gravity theory living in an extra dimension of space-time. In fact, parameters, fields and different processes in the gauge theory are translated into appropriate equivalent on the gravity side. For instance, the (thermal) vacuum state on the gauge theory side corresponds to the (black hole-AdS) pure AdS in the gravity theory. Thermalization process, which generally means evolution of a state from zero temperature to a thermal state, is dual to black hole formation in the gravity theory [6]. Moreover, as another example, the meson, quark-antiquark bound state, living in the QGP can be identified with a classical string in the gravity and by using the expectation value of the Wilson loop the static potential between a quark and antiquark has been firstly found in [7]. For more information, the interested reader is referred to [1] and references therein. 
In this paper, we consider a probe stable meson in the QGP at zero temperature and chemical potential. Then the temperature and the chemical potential are simultaneously raised from zero to finite values $T_{f}$ and $\mu_{f}$, respectively. Now the questions we would like to answer are how the stable meson reacts to the energy injection into the system and what the characteristics of the new meson state are? Furthermore, it is instructive to know how much time is needed for the meson to fall into the final excited state, i.e. excitation time, and what the effect of the final values of the temperature and the chemical potential is on the excitation time? By excited state we mean a meson which has a higher energy than the ground state, i.e. the meson in the initial static gauge theory. The holographic dual of the above system is described by the dynamics of the classical string, with appropriate initial and boundary conditions, in the Reissner-Nordström-AdS Vaidya (RN-AdS-Vaidya) background, as we will review in the next section.

\section{Review on the backgrounds}

The gauge-gravity duality proposes a promising approach to investigate different properties of the strongly coupled field theory. Since we want to study the QGP as a strongly coupled system in the presence of non-zero chemical potential, we firstly review its corresponding holographic dual, i.e. RNAdS background. We then extend our problem to the timedependent case, i.e. RN-AdS-Vaidya, which is dual to the thermalization process in the strongly coupled field theory when the temperature and the chemical potential simultaneously increase.

\subsection{RN-AdS black hole background}

Here we introduce charged black hole metric which is asymptotically AdS. Consider the Einstein-Maxwell anti-de Sitter action [8]:

$S=-\frac{1}{16 \pi G^{d+1}} \int d^{d+1} x \sqrt{-g}\left[\mathcal{R}-\hat{F}^{2}+\frac{d(d-1)}{R^{2}}\right]$,

where $G$ is Newton constant, $\mathcal{R}$ is Ricci scalar, $\hat{F}$ is field strength of the $U(1)$ gauge field and $R$ is $\operatorname{AdS}$ radius which we set to be one. The number of spatial directions is $d$ and it relates to the negative cosmological constant as $\Lambda=-\frac{d(d-1)}{2 R^{2}}$. Equations of the motion obtaining from the above action are

$$
\begin{aligned}
& 0=R_{\mu \nu}-\frac{1}{2} g_{\mu \nu}\left(\mathcal{R}-2 \Lambda-\hat{F}^{2}\right)-2 \hat{F}_{\mu \lambda} \hat{F}_{\mu}^{\lambda}, \\
& 0=\frac{1}{\sqrt{-g}} \partial_{\nu}\left(\sqrt{-g} \hat{F}^{\nu \sigma}\right),
\end{aligned}
$$

where $\mu=0, \ldots, d$. The solution of the equations of motion is the RN-AdS metric ${ }^{1}$ which can be written as [8]

$$
\begin{aligned}
d s^{2} & =\frac{1}{z^{2}}\left[-f(z) d t^{2}+\frac{1}{f(z)} d z^{2}+d \vec{x}^{2}\right], \\
f(z) & =1-M z^{d}+Q^{2} z^{2 d-2},
\end{aligned}
$$

and the time component of the gauge field introduced in (6). $M$ and $Q$ are the mass and the charge of the RN-AdS black hole, respectively. In (3), $z$ is radial coordinate and $z=0$ is the AdS boundary. In addition, $(t, \vec{x})$ are the four dimensional coordinates at the boundary. The gauge-gravity duality indicates that the Hawking temperature of the black hole corresponds to the temperature of the QGP. For $d=4$, the temperature of the $\mathrm{RN}-\mathrm{AdS}_{5}$ black hole is

$T=\frac{1}{\pi z_{h}}\left(1-\frac{1}{2} Q^{2} z_{h}^{6}\right)$.

Here $z_{h}$ is the radius of the event horizon, i.e. the smallest root of $f(z)=0$. The relation between $z_{h}, M$ and $Q$ is

$M=\frac{1}{z_{h}^{4}}+Q^{2} z_{h}^{2}$

Moreover, the time component of the gauge field is given by [8]

$A_{t}=-\frac{\sqrt{3}}{2} Q z^{2}+\Phi$

where $\Phi$ is a constant which plays the role of the electrostatic potential between the boundary of the bulk and the event horizon. It can be defined by applying $A_{t}\left(z_{h}\right)=0$. Thus we obtain

$\Phi=\frac{\sqrt{3}}{2} Q z_{h}^{2}$

Gauge-gravity duality provides a correspondence between the time component of gauge field at the boundary and the chemical potential in dual boundary gauge theory $[8,11]$, i.e.

$\mu=\lim _{z \rightarrow 0} A_{t}=\frac{\sqrt{3}}{2} Q z_{h}^{2}$,

in the AdS radius unit. Hence, it is easy to find that

$\frac{\mu}{T}=\frac{\sqrt{3} \pi}{2} \frac{Q z_{h}^{3}}{1-\frac{1}{2} Q^{2} z_{h}^{6}}$.

\footnotetext{
${ }^{1}$ Holographic models of strongly coupled matter at finite density in equilibrium can display instabilities when $T$ becomes sufficiently small, for instance, see the paper on the RN-AdS black holes in five dimensions where an instability is triggered by a tachyonic field coupled to the sysytem in $\mathcal{N}=8$ gauged supergravity $[9,10]$.
} 


\subsection{RN-AdS-Vaidya background}

Let's generalize the static background (3) to the timedependent case. It can be achieved by adding external source terms to the action (1). Then, the equations of motion for general $d$ are [8]

$$
\begin{aligned}
& 8 \pi G^{d+1} T_{\mu \nu}^{(e x t)}=R_{\mu \nu}-\frac{1}{2} g_{\mu \nu}\left(\mathcal{R}-2 \Lambda-\hat{F}^{2}\right)-2 \hat{F}_{\mu \lambda} \hat{F}_{\nu}^{\lambda}, \\
& 8 \pi G^{d+1} J_{(e x t)}^{\mu}=\frac{1}{\sqrt{-g}} \partial_{\nu}\left(\sqrt{-g} \hat{F}^{\nu \mu}\right) .
\end{aligned}
$$

The RN-AdS-Vaidya metric, in the Eddington-Finkelstein coordinates, is then given by

$$
\begin{aligned}
d s^{2} & =\frac{1}{z^{2}}\left[-F(\bar{v}, z) d \bar{v}^{2}-2 d z d \bar{v}+d \vec{x}^{2}\right], \\
F(\bar{v}, z) & =1-M(\bar{v}) z^{d}+Q(\bar{v})^{2} z^{2 d-2}, \\
A_{\mu} & =-\frac{Q(\bar{v})}{c} z^{d-2} \delta_{\mu \bar{v}},
\end{aligned}
$$

provided that

$$
\begin{aligned}
& 8 \pi G^{d+1} T_{\mu \nu}^{(e x t)} \\
& \quad=z^{d-1}\left[\frac{(d-1)}{2} \dot{M}(\bar{v})-(d-1) z^{(d-2)} Q(\bar{v}) \dot{Q}(\bar{v})\right] \delta_{\mu \bar{v}} \delta_{\nu \bar{v}}, \\
& 8 \pi G^{d+1} J_{(e x t)}^{\mu}=\sqrt{\frac{(d-1)(d-2)}{2}} z^{d+1} \dot{Q}(\bar{v}) \delta^{\mu z},
\end{aligned}
$$

where $\dot{Q}(\bar{v})=d Q / d \bar{v}$ and so on. The $\bar{v}$-coordinate reduces to $t$ at the boundary, i.e. $t=\left.\bar{v}\right|_{z=0}$, the time coordinate of the gauge theory. $M(\bar{v})$ and $Q(\bar{v})$ are arbitrary functions that represent how and at what rate the mass and the charge of the RN-AdS black hole increase. Various functions for $M(\bar{v})$ and $Q(\bar{v})$ have been discussed in the literature and it seems that the physical results are independent of the form of the functions [12]. Therefore, we choose

$\mathcal{I}(\bar{v})=\mathcal{I}_{f} \begin{cases}0 & \bar{v}<0, \\ k^{-1}\left[\bar{v}-\frac{k}{2 \pi} \sin \left(\frac{2 \pi \bar{v}}{k}\right)\right] & 0 \leqslant \bar{v} \leqslant k, \\ 1 & \bar{v}>k,\end{cases}$

where $\mathcal{I} \in(M, Q)$. The transition time $k$ is the time interval that the mass (charge) of the black hole needs to reach its final value $M_{f}\left(Q_{f}\right)$. For $k \ll 1(k \gg 1)$ the transition time is small (large) which is usually called fast (slow) quench. According to the gauge-gravity duality, study of the $\mathrm{RN}-\mathrm{AdS}$-Vaidya metric on the gravity side corresponds to the study of thermalization of strongly coupled QGP in the presence of the chemical potential in the gauge theory. Note that the relation between $M_{f}, Q_{f}$ and $z_{h}$ is still given by (5).

\section{Expectation value of Wilson loop}

The static potential between a quark and anti-quark has been extensively studied, for example see [1,13-15]. In this section, we want to review how this static potential can be obtained from the duality point of view. To do so, we use the expectation value of the Wilson loop as a gauge invariant operator. Specifically, on the one hand, we use the time-like Wilson loop in rectangular form $\mathcal{C}$. One side of rectangle, $l$, is spatial corresponding to the distance between quarkantiquark pair and the other side is temporal, $\mathcal{T}$. If we assume that $\mathcal{T} \gg l$, meaning that the world-sheet is translationally invariant along the time direction, the expectation value of the Wilson loop is [1]

$\langle W(\mathcal{C})\rangle=e^{-i(2 m+V(l)) \mathcal{T}}$,

where $m$ is the rest mass of quark (antiquark) and $V(l)$ represents the static potential energy between the pair. On the other hand, the gauge-gravity duality proposes that in order to calculate the static potential in the QGP at finite chemical potential, one needs to probe the RN-AdS black hole geometry by the classical string $[16,17]$. In other words, the expectation value of the Wilson loop, in the saddle point approximation, is dual to the on-shell action of classical open string that its end points are located on the boundary with distance $l$. Therefore,

$\langle W(\mathcal{C})\rangle=e^{i S(\mathcal{C})}$,

where $S(\mathcal{C})$ is value of the Nambu-Goto action

$S=\frac{-1}{2 \pi \alpha^{\prime}} \int d \tau d \sigma \sqrt{-\operatorname{det}\left(g_{a b}\right)}$.

on the rectangle $\mathcal{C}$. The tension of string is proportional to $\alpha^{\prime-1} \equiv l_{s}{ }^{-2}$ and $l_{s}$ is the fundamental length scale of string. Also, $\tau$ and $\sigma$ parametrize the two dimensional string worldsheet. $g_{a b}=G_{\mu \nu} \frac{\partial X^{\mu}}{\partial \xi^{a}} \frac{\partial X^{v}}{\partial \xi^{b}}$ is the induced metric on the worldsheet. Here $G_{\mu \nu}$ and $X^{\mu}\left(\xi^{a}=\tau, \sigma\right)$ are the metric and the bulk (world-sheet) coordinates, respectively. This action, in fact, describes the dynamics of the classical string in any desired geometry. As we will see, using (14)-(16), the static potential can be found. Note that, the rest mass $m$ is equal to $\frac{\sqrt{\lambda}}{2 \pi} \int_{\epsilon}^{z h} \frac{d z}{z^{2}}[1]$, where $\epsilon$ is IR regulator in the gravity theory and according to UV/IR connection, it corresponds to the UV cut-off in the gauge theory.

In order to calculate the $S(\mathcal{C})$ in the RN-AdS black hole background (3), we parametrize the two-dimensional worldsheet of the string as $\tau=t, \sigma=x_{3} \equiv x$. All bulk coordinates, except $z$ and $x$, are chosen to be constant and therefore the shape of the string is described by $z=z(x)$. Hence the action (16) reduces to

$S=-\frac{\mathcal{T}}{2 \pi \alpha^{\prime}} \int_{-\frac{l}{2}}^{\frac{l}{2}} d x \frac{1}{z^{2}} \sqrt{z^{\prime 2}+f(z)}$, 
where $z^{\prime}=d z / d x$. Since the Lagrangian does not depend explicitly on $x$, the associated Hamiltonian is a constant of motion. After some simple algebra, we get

$z^{\prime}(x)= \pm \frac{z_{*}^{2} f(z)}{\sqrt{f\left(z_{*}\right)} z^{2}} \sqrt{1-\frac{f\left(z_{*}\right)}{f(z)}\left(\frac{z}{z_{*}}\right)^{4}}$

where $z=z_{*}$ at $z^{\prime}(x)=0$. Using the new coordinate $y=$ $z_{*} / z$ and the explicit form of $f(z)$, it turns out

$$
\begin{aligned}
& \int_{ \pm \frac{l}{2}}^{0} d x=\mp z_{*} \sqrt{1-y_{h}^{4}+Q_{f}^{2} z_{*}^{6}\left(1-y_{h}{ }^{-2}\right)} \\
& \quad \times \int_{1}^{\infty} \frac{d y}{\sqrt{\left[y^{4}-1+Q_{f}^{2} z_{*}{ }^{6}\left(y^{-2}-1\right)\right]\left[y^{4}-y_{h}^{4}+Q_{f^{2} z_{*}^{6}\left(y^{-2}-y_{h}^{-2}\right)}\right.}},
\end{aligned}
$$

where $y_{h}=z_{*} / z_{h}$. The on-shell action can be then found by inserting (18) and (19) into (17). Furthermore, by applying (14) and (15) the potential energy between the quark and anti-quark will be obtained that is in agreement with results reported in [18]. Notice that, in order to get a finite value for the potential energy, we need to subtract the rest mass of the quarks from the on-shell action and we therefore have

$$
\begin{aligned}
V= & \frac{1}{\pi \alpha^{\prime}}\left[\frac{1}{z_{*}} \int_{1}^{\infty} d y\left(\sqrt{\frac{y^{4}-y_{h}^{4}+Q_{f}^{2} z_{*}^{6}\left(y^{-2}-y_{h}^{-2}\right)}{y^{4}-1+Q_{f}^{2} z_{*}^{6}\left(y^{-2}-1\right)}}-1\right)\right. \\
& \left.-\left(\frac{1}{z_{*}}-\frac{1}{z_{h}}\right)\right] .
\end{aligned}
$$

Before closing this part, two points should be noted. First, as we will see, the Eq. (19) will be utilized as an initial condition for the time evolution of the classical string in the RN-AdSVaidya background. Second, in the next section, we observe that the expectation value of the time-dependent Wilson loop oscillates around the static potential resulted in (20).

Now our goal is to calculate the evolution of expectation value of Wilson loop in the RN-AdS-Vaidya geometry. In this geometry, the two-dimensional world-sheet of the string is no longer translationally invariant in the time direction. Thus the condition $\mathcal{T} \gg l$ does not valid and therefore in this case, the expectation value of the Wilson loop (14) can be written as

$$
\langle W(\mathcal{C})\rangle=e^{-i \int d t \mathcal{W}(t)},
$$

where, using the gauge-gravity duality, $\mathcal{W}(t)$ is the on-shell action of string without integrating over $t$-coordinate. It is important to note that although $\mathcal{W}$ is a function of different parameters in the gauge theory, such as temperature, chemical potential and distance $l$, we only show its time dependence, explicitly. As before, $\mathcal{W}(t)$, or equivalently the onshell string action, diverges and in order to regularize this divergence, similar to the static case, we have

$$
\mathcal{W}_{R}(t)=\mathcal{W}(t)-2 m \equiv \int d \sigma\left(\sqrt{-\operatorname{det}\left(g_{a b}\right)}\right)_{\text {on-shell }}-2 m
$$

The subscript " $R$ " in (22) refers to the regularized version of $\mathcal{W}(t)$. To calculate the string on-shell action, similar to [1921 ], we choose the null coordinates $(u, v)$ to parametrize the two-dimensional world-sheet of the string and therefore all the coordinates on the world-sheet depend on $u$ and $v$. Then we choose the following ansatz:

$\bar{v}=V(u, v), \quad z=Z(u, v), \quad x_{3}=X(u, v)$.

Note that due to rotational symmetry in spatial coordinates, there is no difference among them. Substituting this ansatz into the Nambo-Goto action (16), the equations of motion can be found. Hence, we finally have

$$
\begin{aligned}
V_{, u v}= & \left(\frac{F_{, Z}}{2}-\frac{F}{Z}\right) V_{, u} V_{, v}+\frac{1}{Z} X_{, u} X_{, v}, \\
Z_{, u v}= & \left(\frac{F^{2}}{Z}-\frac{F}{2} F_{, Z}-\frac{1}{2} F_{, V}\right) V_{, u} V_{, v} \\
& +\left(\frac{F}{Z}-\frac{F_{, Z}}{2}\right)\left(Z_{, u} V_{, v}+Z_{, v} V_{, u}\right) \\
& +\frac{2}{Z} Z_{, u} Z_{, v}-\frac{F}{Z} X_{, u} X_{, v}, X_{, u v}=\frac{Z_{, u} X_{, v}+Z_{, v} X_{, u}}{Z},
\end{aligned}
$$

where, for an arbitrary function $A(a, b)$, we define $A_{, a}=\frac{\partial A}{\partial a}$ and $A_{, a b}=\frac{\partial^{2} A}{\partial a \partial b}$. Since $u$ and $v$ are null coordinates, we need to impose two constraints corresponding to $g_{u u}=0$ and $g_{v v}=0$. Thus, two constraint equations turn out to be

$$
\begin{aligned}
& C_{1}=\frac{1}{Z^{2}}\left(F V_{, u}^{2}+2 V_{, u} Z_{, u}-X_{, u}^{2}\right)=0, \\
& C_{2}=\frac{1}{Z^{2}}\left(F V_{, v}^{2}+2 V_{, v} Z_{, v}-X_{, v}^{2}\right)=0 .
\end{aligned}
$$

In order to solve the equations of motion (24) subject to constraint equations (25), the suitable boundary and initial conditions are essential. In the Appendices B and C, we obtain the appropriate boundary and initial conditions, respectively. After solving the equations of motion (24), one can easily find the time evolution of $\mathcal{W}_{R}(t)$ when the other parameters are kept fixed.

\section{Numerical results}

As we have already mentioned and shown by the Figs. 1, 2 , 3 and 4 the expectation value of the Wilson loop oscillates around the static potential. In fact, before injecting energy $(t<0)$, the quark-antiquark pair is in ground state. However, as the energy injection is started, the temperature and the chemical potential increases and therefore the pair is excited. When the energy injection ceases, the pair falls into a final 


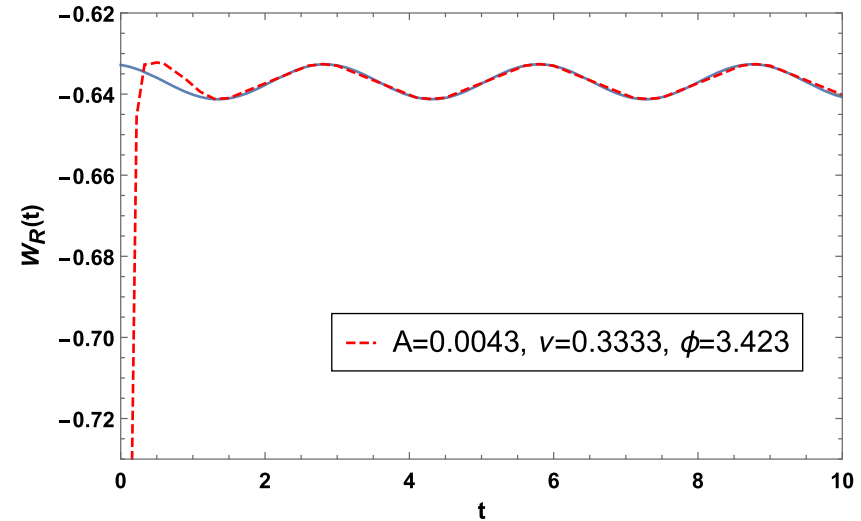

Fig. $1 \mathcal{W}_{R}(t)$ in terms of the boundary time $t$ for $l=1, k=0.3$ and $\mu_{f} / T_{f}=3.15$. For the left (right) graph $\mu_{f}=0.3188$ (0.3925) and $T_{f}=0.1012(0.1246)$. The static potentials for left (right) graph is

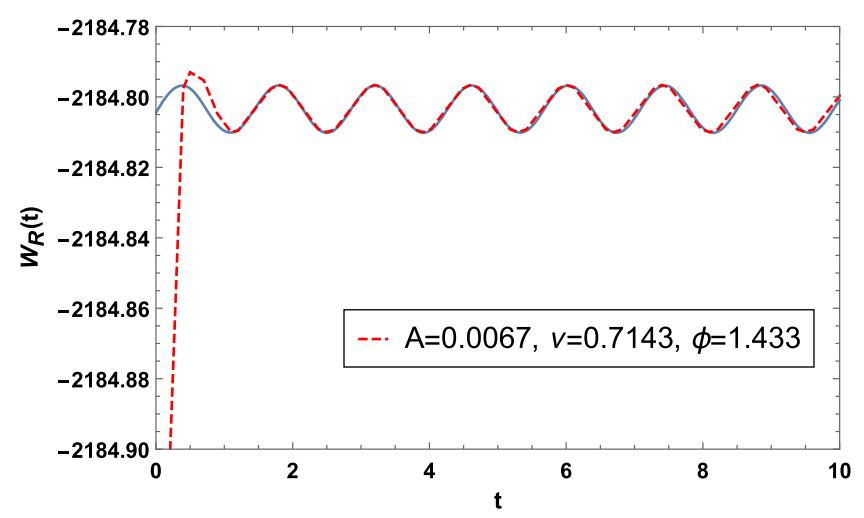

Fig. $2 \mathcal{W}_{R}(t)$ in terms of boundary time $t$ for $k=0.3, \mu_{f}=0.4503$, $T_{f}=0.1428$ and $\mu_{f} / T_{f}=3.15$. The distance between the pair is $l=0.5(l=1)$ for the left (right) graph. The static potential is

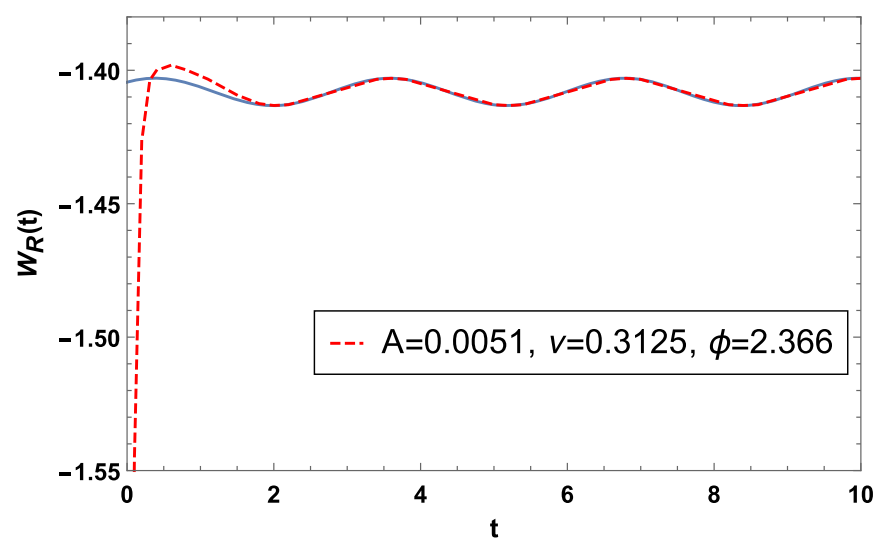

Fig. $3 \mathcal{W}_{R}(t)$ in terms of the boundary time $t$ for $l=1, k=0.3$ and $\mu_{f} / T_{f}=0.1$. For the left (right) graph $\mu_{f}=0.0134$ (0.0220) and $T_{f}=0.1340(0.2200)$. The static potentials for left (right) graph is

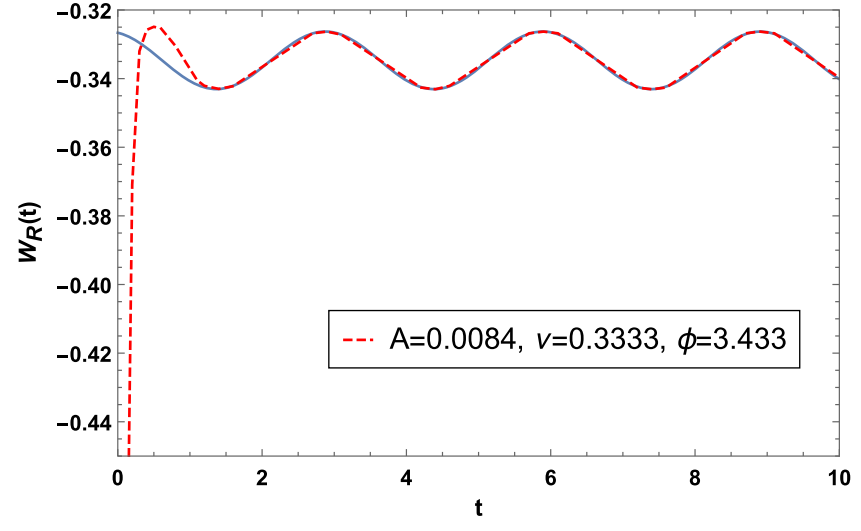

$V=-0.6369(-0.3347)$. The dashed red curve shows our numerical results and the blue sine curve is the fitted function (26)

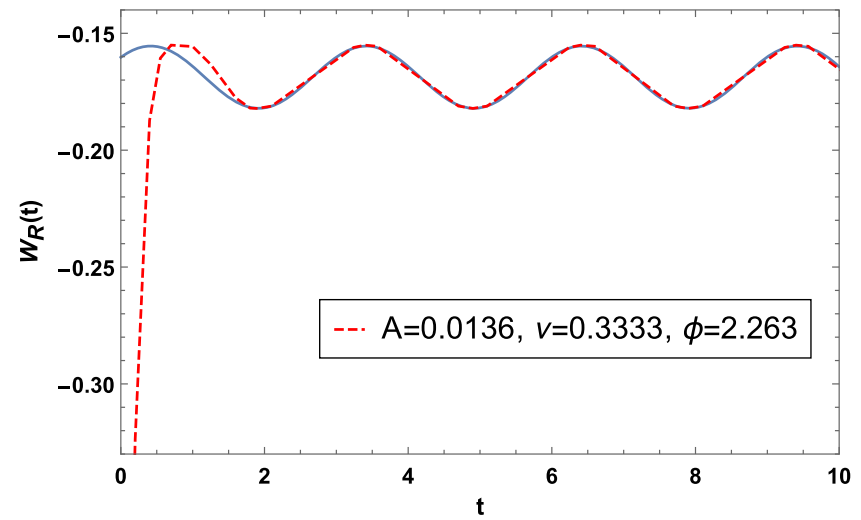

$V(l)=-2184.8(-0.1686)$ for left (right) graph. The dashed red curve shows our numerical results and the blue sine curve is the fitted function (26)

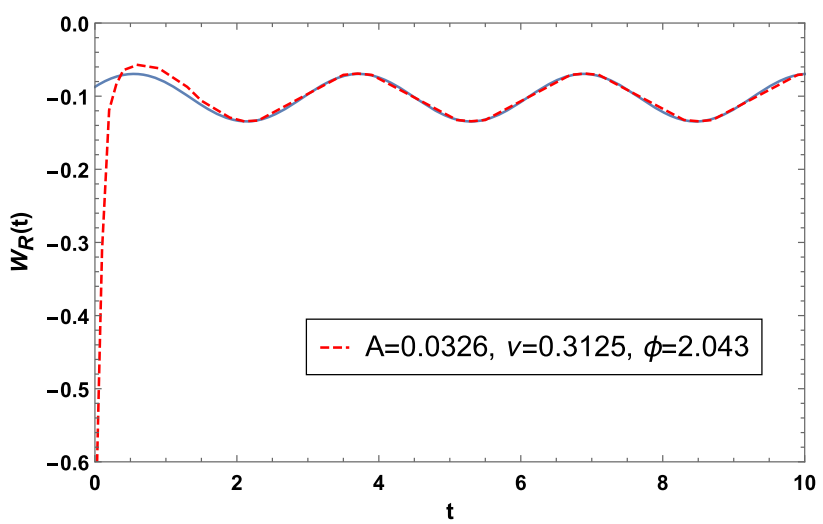

$V=-1.4081(-0.1017)$ respectively. The dashed red curve shows our numerical results and the blue sine curve is the fitted function (26) 


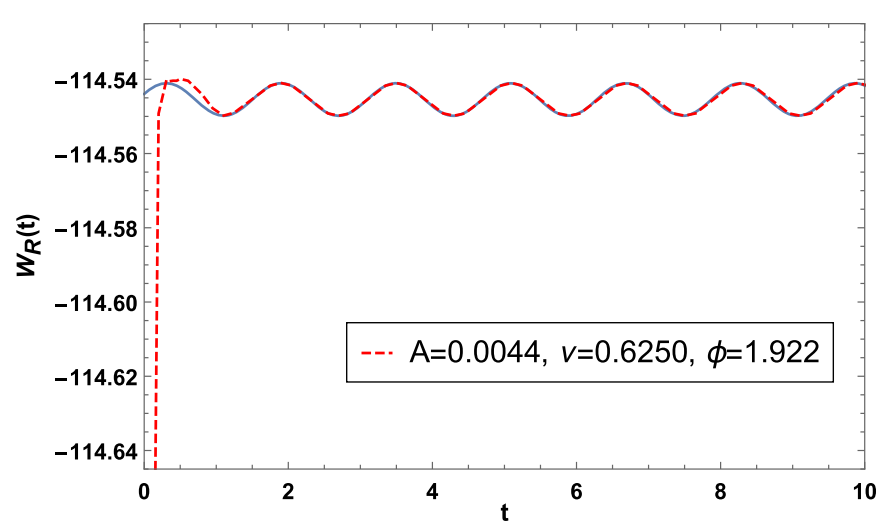

Fig. $4 \mathcal{W}_{R}(t)$ in terms of boundary time $t$ for $k=0.3, \mu_{f}=0.0220$, $T_{f}=0.2200$ and $\mu_{f} / T_{f}=0.1$. The distance between the pair is $l=0.5(l=1)$ for the left (right) graph. The static potential is

excited state with specific frequency and amplitude of oscillation. In other words, these oscillations can be interpreted as if the energy injection puts the meson in the excited state [1921]. As a consequence of conformal invariance at finite temperature, there are two independent parameters, say $\mu_{f} / T_{f}$ and $l T_{f}$. We are now interested in investigating the effect of these parameters on the characteristic of oscillation, i.e. frequency and amplitude. Thus our results are classified into the following categories:

\section{- $\frac{\mu_{f}}{T_{f}}>1$ and fixed}

In Fig. 1, we show how the expectation value of Wilson loop evolves with time for $l T_{f}=0.1012$ (left panel) and $l T_{f}=0.1246$ (right panel) for fixed $\mu_{f} / T_{f}=3.15$. We observe that by raising the final value of the temperature of the QGP while the distance between the quark and antiquark is kept fixed, the amplitude of the oscillation increases, also (the energy of the excited pair increases based on the relation between amplitude and energy in simple harmonic oscillator). However, the value of the frequency is independent of the final temperature in agreement with the numerical results of [22]. In Fig. 2, we have $l T_{f}=0.0714$ (left panel) and $l T_{f}=0.1428$ (right panel) for fixed $\mu_{f} / T_{f}=3.15$. In this figure with increasing the distance between the quark and antiquark while the temperature is kept fixed, the amplitude (frequency) of oscillation increases (decreases).

- $\frac{\mu_{f}}{T_{f}}<1$ and fixed

All results for this case are similar to the previous case. In other words, it seems that in the case at hand the value of $\mu_{f} / T_{f}$ does not change the behavior of the expectation value of the Wilson loop, qualitatively.

An interesting point we would like to emphasize here is that since we are working in the probe limit, the energy of the

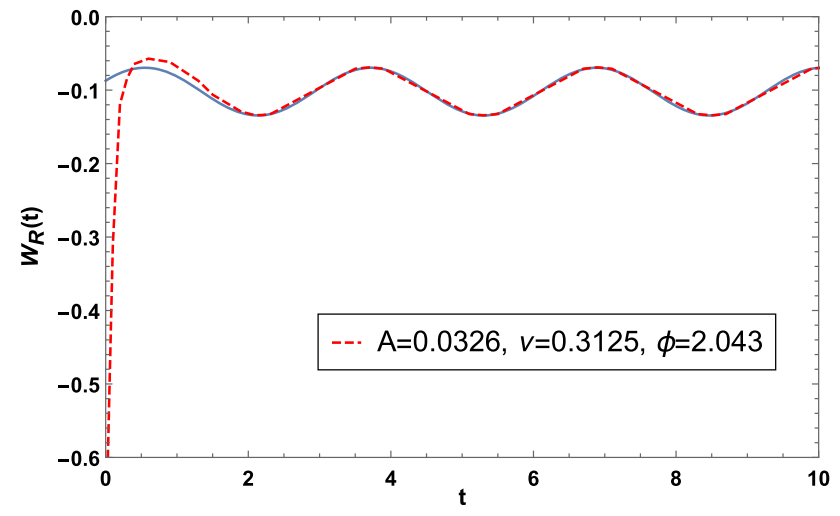

$V(l)=-114.55(-0.1017)$ for left (right) graph. The dashed red curve shows our numerical results and the blue sine curve is the fitted function (26)

meson does not dissipate in the plasma and therefore the oscillation remains unchanged.

Up to now, we investigate the response of the system to the time-dependent change in the temperature and the chemical potential. In fact, the response of the system is described by the behavior of the expectation value of the Wilson loop, $\mathcal{W}_{R}(t)$, in terms of boundary time. We observe that the expectation value oscillates around the static potential with a specific value of frequency, $v$, and amplitude of oscillation, $A$. The characteristics of oscillation depend on the final values of the chemical potential and the temperature as well as the distance between the pair. Now, the question we would like to answer is, at which time the expectation value of the Wilson loop starts oscillating around the static potential? And we call it excitation time. To do so, we define the following function:

$\mathcal{W}_{f}(t)=A \cos (2 \pi v t+\phi)$,

where $A, v$ and $\phi$ can be found from $\mathcal{W}_{R}(t)$ at asymptotic times. We then define a time-dependent function

$$
\begin{aligned}
& \epsilon_{\mu}(t)=\left|\frac{\mathcal{W}_{R}(t)-\mathcal{W}_{f}(t)}{\mathcal{W}_{R}(t)}\right|_{T, l}, \\
& \epsilon_{T}(t)=\left|\frac{\mathcal{W}_{R}(t)-\mathcal{W}_{f}(t)}{\mathcal{W}_{R}(t)}\right|_{\mu, l}, \\
& \epsilon_{l}(t)=\left|\frac{\mathcal{W}_{R}(t)-\mathcal{W}_{f}(t)}{\mathcal{W}_{R}(t)}\right|_{\mu, T},
\end{aligned}
$$

where in (27a) $T$ and $l$ are kept fixed while $\mu$ changes and so on. Thus the excitation time, $t_{e x}$, is defined as the time which satisfies $\epsilon\left(t_{e x}\right)<5 \times 10^{-6}$ and $\epsilon(t)$ stays below this limit afterwards. $^{2}$

\footnotetext{
2 One can choose the other relevant numbers and see the final results does not change qualitatively.
} 

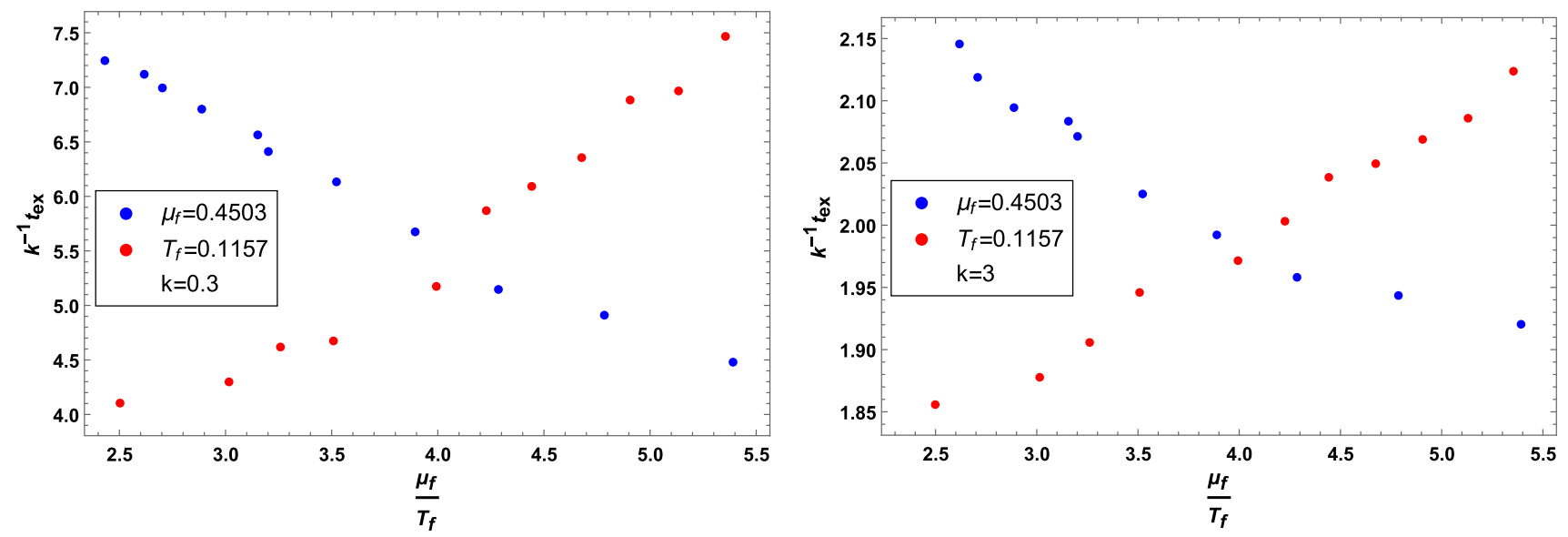

Fig. 5 The excitation time in terms of $\mu_{f} / T_{f}$ for $l=1$

In Fig. 5 (left), blue points, the final temperature of the system decreases while the other parameters contributing to the time evolution of the expectation value are kept fixed. As it is clearly seen, at higher temperatures the meson falls into the excited state later. In other words, the higher temperature, the larger (rescaled) excitation time, $\left(k^{-1}\right) t_{e x}$. This result can be intuitively understood: at higher temperatures the thermal fluctuations become more significant i.e. in any system with non-zero temperature, the thermal fluctuations are present and as the temperature increases, the thermal fluctuations become more highlight. In other words, the particles gain more kinetic energy and bounce more frequently off of one another, so that can lead to more deviation of the (state of) particles from their steady state. Thus this effect prevents the meson from sooner fall. In the same figure, red points, since we would like to investigate the effect of the chemical potential on the excitation time, the chemical potential is varied and the other parameters are kept fixed. Similar to the previous case, this figure indicates that the rescaled excitation time and chemical potential increase with respect to each other. As a matter of fact, for larger values of the temperature and the chemical potential, the rescaled excitation time becomes larger. In short, the effect of temperature on the rescaled excitation time is similar to the chemical potential. Apart from the fast quench, in the right panel, the rescaled excitation time is plotted as a function of $\mu_{f} / T_{f}$ for the case of the slow quench, i.e. $k=3$. It is obviously seen that in both cases the behavior of excitation times is the same. However, $k^{-1} t_{e x}$ is larger for the case of the fast quench.

In Fig. 6, the distance $l$ is increased while the other parameters are kept fixed. In contrast to the chemical potential and the temperature, the distance is an intrinsic characteristic of the meson in the plasma. This figure shows that for larger value of distance $l$, meaning that the meson is less stable, the excitation time increases. Here, by stability we mean that the value of the static potential is lower at zero temperature and chemical potential, or equivalently before the energy

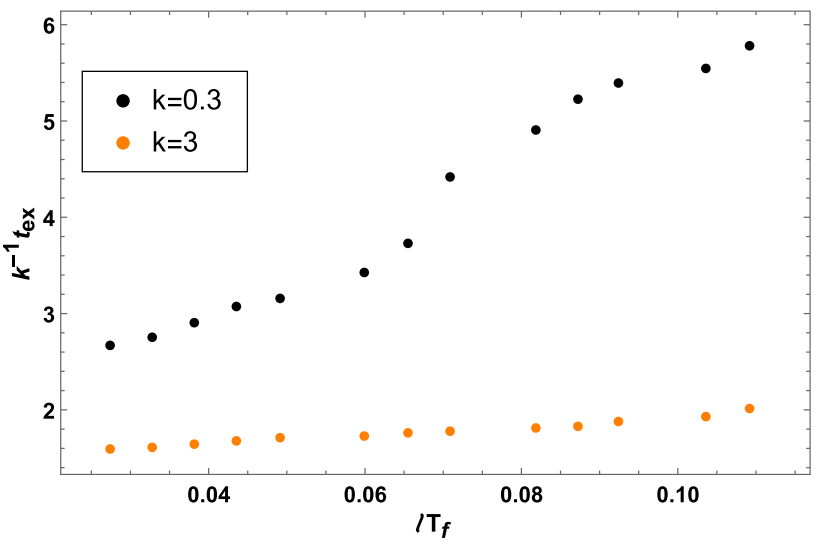

Fig. 6 The excitation time in terms of $l T_{f}$ for $T_{f}=0.1091$ and $\mu_{f}=$ 0.4811

injection, for smaller values of distance $l$. Although for both quenches the rescaled excitation time behaves similarly, the value of $k^{-1} t_{e x}$ is larger for the fast quench. In other words, this figure indicates that the meson with smaller $l$, i.e. the more stable quark-antiquark bound states are excited sooner. It may be related to the screening of the force between color charges of the quark and antiquark due to the presence of the medium since they, quark and antiquark, can not communicate, easily. Notice that the distance between quark and antiquark can not be too large because the meson will then dissociate in the plasma.

\section{Conclusions}

Our main outcomes can be summarized as follows:

- We observe that the expectation value of the timedependent Wilson loop oscillates around the static potential. It may be interpreted as the string connecting quark and antiquark (or flux tube resulted from the gluon fields 
between the pair) in field theory with Dirichlet boundary conditions on the endpoints. After the energy injection, the string is oscillating in one of the its normal modes or equivalently the quark-antiquark bound state has been excited. As a matter of fact, we investigate the effect of the chemical potential and the temperature on this normal mode.

- Making larger each parameter under study in this paper, i.e. final chemical potential, final temperature and the quark-antiquark distance, the energy of the bound state, based on classical harmonic oscillator model, increases since the amplitude of the oscillation becomes larger.

- The oscillation frequency is independent of the temperature and approximately of the chemical potential. It is intuitively comprehensible. Similar to the classical harmonic oscillator, the oscillation frequency is an intrinsic characteristic of the meson and is independent of the environmental changes. However, the larger distance between quark and antiquark, the smaller oscillation frequency.

- Consider a meson in the plasma with non-zero temperature and chemical potential. For larger values of the chemical potential and higher temperatures, the excitation time of the meson increases. In other words, when the plasma is hotter or denser the meson falls into the final excited state more slowly.

- Consider a plasma where its temperature and chemical potential are kept fixed. Then the more stable meson at $T=\mu=0$, corresponding to the smaller distance between quark and antiquark, falls into the final excited state sooner.

- All of the above results are confirmed for the slow and the fast quench. By slow (fast) quench we mean the energy injection into the system is done slowly (rapidly).

We would like to emphasize that, apart from the first result in the third item, the other outcomes can not be argued by comparing to the simple harmonic oscillator. In the case of the second item, one expects that while the energy is injected into the system by an external source the energy of the bound state increases. On the other hand, since our results indicate that the amplitude of the oscillation always increases after the energy injection, this reminds us of the relation between the energy and amplitude of the oscillation in the simple harmonic oscillator and we therefore conclude that the energy of the meson increases in the plasma. However, it is important to notice that this relation is not as easy as the simple harmonic oscillator, i.e. (energy) $\propto$ (amplitude) $)^{2}$, as shown by our numerical results. In other words, the amplitude of oscillation, in fact, is a complicated and non-linear function of the different variables such as temperature, chemical potential and timescale of the energy injection.

In addition, studying non-local observables [8,23] such as the two-point correlation function and the expectation value of Wilson loop as probes of thermalization process, show that the larger $\mu / T$ the larger thermalization time. However, by studying the two-point correlation function, the expectation value of Wilson loop and the entanglement entropy [24], the results indicate that for a fixed small value of $l T(l T \ll 1)$ and small values of $\mu / T$ the thermalization time decreases by increasing $\mu / T$, thus plasma thermalizes faster. But, for larger values of $\mu / T$ the thermalization time increases with increasing $\mu / T$. Also, by increasing the value of $l T$ this nonmonotonic behavior becomes less pronounced and finally disappears, so that a monotonic behavior has been observed for $l T \gg 1$.

More recently, equilibration of a dynamical scalar operator is considered in the charged QGP during its equilibration [25]. The numerical outcomes show that the equilibration time can be a decreasing or increasing function of $\mu / T$ and depends on the energy injection, so that one cannot report the general behavior. Furthermore, in [26] studying non-hydrodynamic quasinormal modes of a scalar field show that the equilibration time decreases with increasing the chemical potential far from the critical point, while close to the critical point it would increase. On the other hand, the thermalization time always increases with increasing the chemical potential. In summary, although the excitation time increases for larger values of the chemical potential in our case, it should be instructive to check our outcomes in other gauge theories with holographic dual to find a general behavior.

Acknowledgements M. A. would like to thank School of Physics of Institute for research in fundamental sciences (IPM) for the research facilities and environment. We also would like to thank the referee for his/her comments which help us to improve presentation of the manuscript.

Data Availability Statement This manuscript has no associated data or the data will not be deposited. [Authors' comment: Since the paper provides a qualitative explanation, we do not use date in this paper.]

Open Access This article is distributed under the terms of the Creative Commons Attribution 4.0 International License (http://creativecomm ons.org/licenses/by/4.0/), which permits unrestricted use, distribution, and reproduction in any medium, provided you give appropriate credit to the original author(s) and the source, provide a link to the Creative Commons license, and indicate if changes were made.

Funded by $\mathrm{SCOAP}^{3}$.

\section{Appendix A: Large chemical potential limit}

In this section, we study the potential of quark-antiquark when the charge $Q$ or equivalently the chemical potential $\mu$ is large enough so the temperature $T$ can be neglected. In this limit, using (4) and (5), we have:

$Q^{2}=2 z_{h}^{-6}$, 


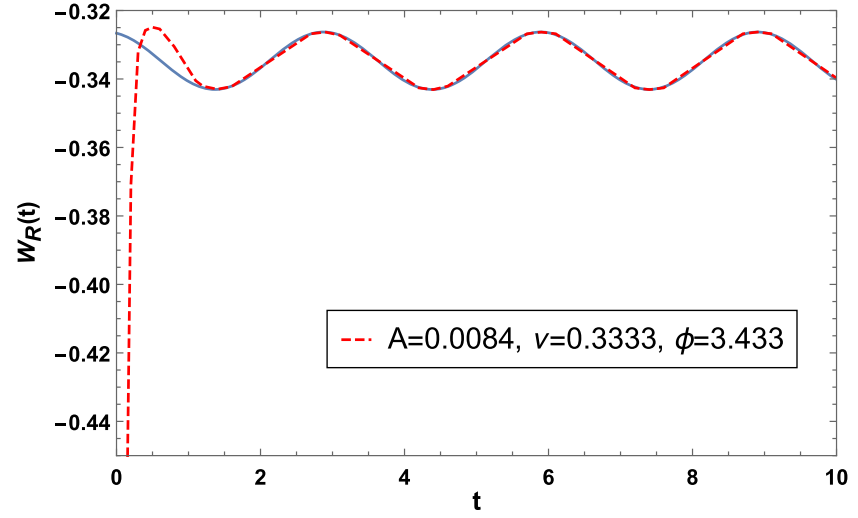

Fig. $7 \mathcal{W}_{R}(t)$ in terms of boundary time $t$ for $k=0.3$ and $l=1$. For the left panel $\mu_{f}=0.3925, T_{f}=0.1246$ and for the right panel $\mu_{f}=$ $0.6981, T_{f}=0$. The static potential is $V(l)=-0.3347(-0.3912)$

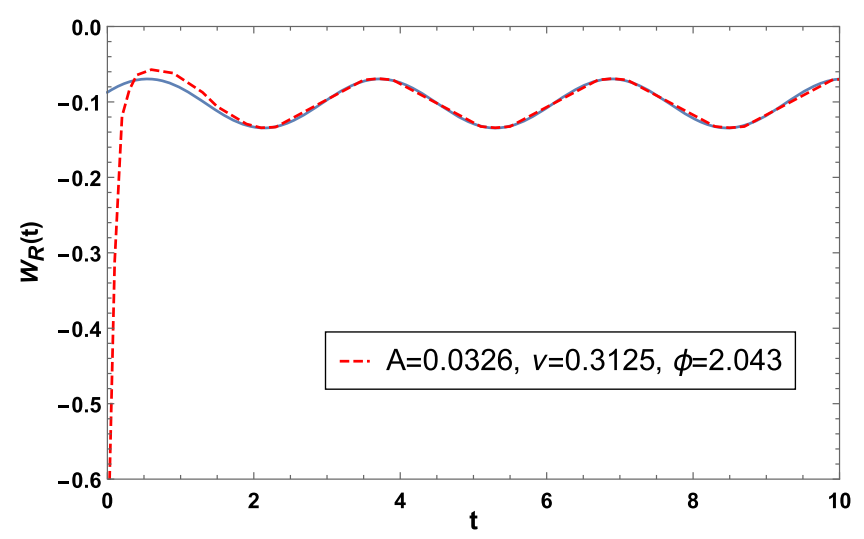

Fig. $8 \mathcal{W}_{R}(t)$ in terms of boundary time $t$ for $k=0.3$ and $l=1$. For the left panel $\mu_{f}=0.0220, T_{f}=0.2200$ and for the right panel $\mu_{f}=$ $0.8475, T_{f}=0$. The static potential is $V(l)=-0.1017(-0.1793)$

$$
M=3 z_{h}^{-4}
$$

and thus, by using (8), one can find

$\mu=\sqrt{\frac{3}{2}} z_{h}^{-1}$.

In Fig. 7 (left panel) we set $\mu_{f} / T_{f}=3.15$. However, in the right panel $T_{f}$ is zero, i.e. large chemical potential. We see that the static potential of the pair decreases and the amplitude of the oscillation increases. But, the frequency of oscillation does not change. In Fig. 8, we have $\mu_{f} / T_{f}=0.1$ for the left panel and large $\mu$ for the right panel. The results are in complete agreement with the Fig. 7 . In addition, note that these results show that, in the large $\mu$ limit, by increasing the chemical potential, the final static potential also rises. It is intuitively correct since the matter screens the interaction between the quark and antiquark.

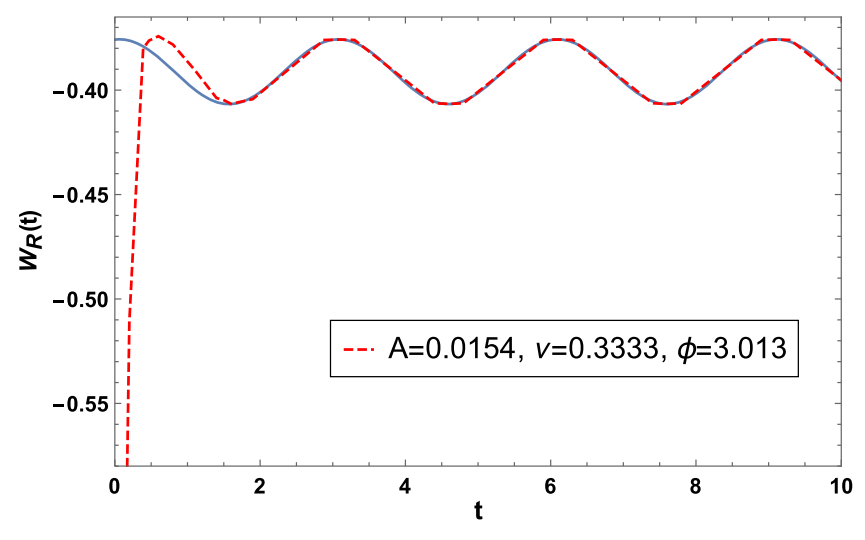

for left (right) graph. The dashed red curve shows our numerical results and the blue sine curve is the fitted function (26)

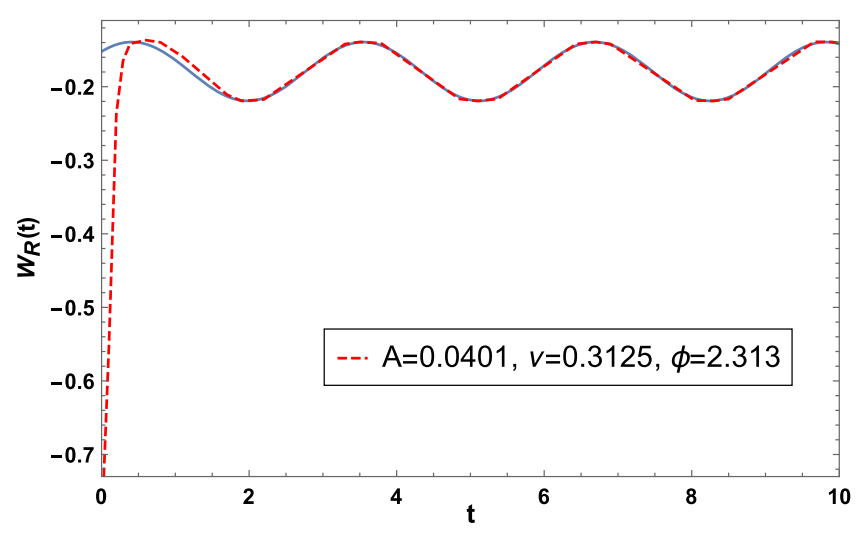

for left (right) graph. The dashed red curve shows our numerical results and the blue sine curve is the fitted function (26)

\section{Appendix B: Boundary condition}

Here, we state the boundary conditions for solving the equations of motion (24). By applying the diffeomorphism invariance on the two-dimensional world-sheet of the string, one of the endpoints of string on the boundary can be fixed to be at $u=v$ and the other one at $u=v+L$. Thus, for the coordinates $Z$ and $X$, the boundary conditions are

$$
\begin{aligned}
&\left.Z\right|_{u=v}=0 ;\left.\quad X\right|_{u=v}=\frac{-l}{2}, \\
&\left.Z\right|_{u=v+L}=0 ;\left.\quad X\right|_{u=v+L}=\frac{l}{2} .
\end{aligned}
$$

In fact the above boundary conditions in the gauge theory means that the distance between the quark and antiquark does not change during the energy injection. Similar boundary conditions in the static case [27] and in the time-dependent case [19-21] have been studied. The other boundary conditions can be obtained by expanding $V(u, v), Z(u, v)$ and 
$X(u, v)$ about the point $u=v$ at the boundary. Therefore, one gets

$$
\begin{aligned}
& V(u, v)=V_{0}(v)+V_{1}(v)(u-v)+\cdots, \\
& Z(u, v)=Z_{1}(v)(u-v)+Z_{2}(v)(u-v)^{2}+\cdots, \\
& X(u, v)=\frac{-l}{2}+X_{1}(v)(u-v)+\cdots
\end{aligned}
$$

The same expansion is done for the other point $u=v+$ $L$ at the boundary. By inserting the above equations into the evolution equations (24) and demanding the regularity condition at $u=v$ and $u=v+L$, one can obtain the remaining boundary conditions. Notice that the results should be consistent with the constraint equations (25). Finally, at the boundary, i.e. $u=v$, conditions are:

$$
\begin{aligned}
V(u, v)= & V_{0}(v)+\mathcal{O}\left((u-v)^{5}\right), \\
Z(u, v)= & \frac{\dot{V}_{0}(v)}{2}(u-v)+\frac{\ddot{V}_{0}(v)}{4}(u-v)^{2} \\
& +\frac{\dddot{V}_{0}(v)}{12}(u-v)^{3}+\mathcal{O}\left((u-v)^{4}\right), \\
X(u, v)= & \frac{-l}{2}+\mathcal{O}\left((u-v)^{3}\right),
\end{aligned}
$$

which indicate that

$$
\left.Z_{, u v}\right|_{u=v}=0,\left.\quad 2 Z_{, u}\right|_{u=v}=\dot{V}_{0}(v),
$$

where $\dot{V}(v)=\frac{d V(v)}{d v}$. The same results are obtained for $u=$ $v+L$. We refer the interested reader to [19-21] for more details.

\section{Appendix C: Initial condition}

Using (18) and the constraint equations (25), we get the initial condition for $Z, V$ and $X$. We set $f(z)=1$ and replace $x$ and $z$ with the capital forms. Considering $V_{, v}>0$ at the boundary, (B5a) and (B5b), it is easy to find that $Z_{, u}>0$ and $Z_{, v}<0$. By imposing $\left.X_{, u}\right|_{Z=0}=\left.X_{, v}\right|_{Z=0}=0$ and the above conditions on the constraint equations (25), we get

$$
\begin{aligned}
& V_{, u}=Z_{, u}\left(-1+\sqrt{1+\left(\frac{d X}{d Z}\right)^{2}}\right), \\
& V_{, v}=Z_{, v}\left(-1-\sqrt{1+\left(\frac{d X}{d Z}\right)^{2}}\right) .
\end{aligned}
$$

Now by taking the derivative of (C1) and (C2) with respect to $v$ and $u$ respectively and noting that $V_{, u v}=V_{, v u}$, it turns out

$$
\left(Z_{, u} \sqrt{1+\left(\frac{d X}{d Z}\right)^{2}}\right)_{, v}=0 .
$$

Replacing $\frac{d X}{d Z}$, from (18), into the above equation we get the initial condition for $Z(u, v)$

$Z_{2} F_{1}\left(\frac{1}{2}, \frac{1}{4} ; \frac{5}{4} ; \frac{Z^{4}}{Z_{*}^{4}}\right)=\phi(u)-\phi(v)$,

where $\phi(y)$ is an arbitrary function and we choose $\phi(y)=$ $y$ [19-21]. The initial condition for $X(u, v)$ is obtained by integrating (18). We then have

$X(u, v)=\frac{l}{2}-\frac{Z^{3}}{3 Z_{*}^{2}}{ }_{2} F_{1}\left(\frac{1}{2}, \frac{3}{4} ; \frac{7}{4} ; \frac{Z^{4}}{Z_{*}^{4}}\right)$.

Finally, the initial condition for $V(u, v)$ can be obtained from (C1) and (C2)

$V(u, v)=-Z\left(1-{ }_{2} F_{1}\left(\frac{1}{2}, \frac{1}{4} ; \frac{5}{4} ; \frac{Z^{4}}{Z_{*}^{4}}\right)\right)+\chi(v)$,

$V(u, v)=-Z\left(1+{ }_{2} F_{1}\left(\frac{1}{2}, \frac{1}{4} ; \frac{5}{4} ; \frac{Z^{4}}{Z_{*}^{4}}\right)\right)+\tilde{\chi}(u)$,

where $\chi$ and $\tilde{\chi}$ are some kind of arbitrary functions. Also, Using (C4) and equalize the above equations, we can get

$\chi(v)=2 \phi(v), \quad \tilde{\chi}(u)=2 \phi(u)$.

One can find more details in [19-21].

\section{References}

1. J. Casalderrey-Solana, H. Liu, D. Mateos, K. Rajagopal, U.A. Wiedemann, Gauge/String Duality, Hot $Q C D$ and Heavy Ion Collisions (Cambridge University Press, Cambridge, UK, 2014). https://doi.org/10.1017/CBO9781139136747. arXiv:1101.0618 [hep-th

2. A. Rothkopf, T. Hatsuda, S. Sasaki, Complex heavy-quark potential at finite temperature from lattice QCD. Phys. Rev. Lett. 108, 162001 (2012). arXiv:1108.1579 [hep-lat]

3. J.M. Maldacena, The large N limit of superconformal field theories and supergravity, Adv. Theor. Math. Phys. 2, 231 (1998) arXiv:hep-th/9711200 [Int. J. Theor. Phys. 38 (1999) 1113]

4. S.S. Gubser, I.R. Klebanov, A.M. Polyakov, Gauge theory correlators from non-critical string theory. Phys. Lett. B 428, 105 (1998). arXiv:hep-th/9802109

5. E. Witten, Anti-de Sitter space and holography. Adv. Theor. Math. Phys. 2, 253 (1998). arXiv:hep-th/9802150

6. P.M. Chesler, L.G. Yaffe, Numerical solution of gravitational dynamics in asymptotically anti-de Sitter spacetimes. JHEP 1407, 086 (2014). arXiv:1309.1439 [hep-th]

7. J.M. Maldacena, Wilson loops in large N field theories. Phys. Rev. Lett. 80, 4859 (1998). arXiv:hep-th/9803002

8. D. Galante, M. Schvellinger, Thermalization with a chemical potential from AdS spaces. JHEP 1207, 096 (2012). arXiv:1205.1548 [hep-th]

9. S.S. Gubser, I. Mitra, Instability of charged black holes in Anti-de Sitter space. Clay Math. Proc. 1, 221 (2002). arXiv:hep-th/0009126

10. R.A. Konoplya, A. Zhidenko, Stability of higher dimensional Reissner-Nordstrom-anti-de Sitter black holes. Phys. Rev. D 78, 104017 (2008). arXiv:0809.2048 [hep-th]

11. R.C. Myers, M.F. Paulos, A. Sinha, Holographic hydrodynamics with a chemical potential. JHEP 0906, 006 (2009). arXiv:0903.2834 [hep-th] 
12. S. Amiri-Sharifi, M. Ali-Akbari, A. Kishani-Farahani, N. Shafie, Double relaxation via AdS/CFT. Nucl. Phys. B 909, 778 (2016). arXiv:1601.04281 [hep-th]

13. A. Brandhuber, N. Itzhaki, J. Sonnenschein, S. Yankielowicz, Wilson loops in the large $\mathrm{N}$ limit at finite temperature. Phys. Lett. B 434, 36 (1998). arXiv:hep-th/9803137

14. S.J. Rey, S. Theisen, J.T. Yee, Wilson-Polyakov loop at finite temperature in large $\mathrm{N}$ gauge theory and anti-de Sitter supergravity. Nucl. Phys. B 527, 171 (1998). arXiv:hep-th/9803135

15. J. Sonnenschein, Stringy confining Wilson loops. PoS tmr 2000, 008 (2000). arXiv:hep-th/0009146

16. S.J. Zhang, E. Abdalla, Holographic thermalization in charged dilaton anti-de Sitter spacetime. Nucl. Phys. B 896, 569 (2015). arXiv:1503.07700 [hep-th]

17. X.X. Zeng, X.M. Liu, W.B. Liu, Holographic thermalization with a chemical potential in Gauss-Bonnet gravity. JHEP 1403, 031 (2014). arXiv:1311.0718 [hep-th]

18. A. Samberg, C. Ewerz, Heavy probes in strongly coupled plasmas with chemical potential. Springer Proc. Phys. 170, 401 (2016). arXiv:1312.5999 [hep-th]

19. T. Ishii, S. Kinoshita, K. Murata, N. Tanahashi, Dynamical meson melting in holography. JHEP 1404, 099 (2014), arXiv:1401.5106 [hep-th]

20. M. Ali-Akbari, F. Charmchi, A. Davody, H. Ebrahim, L. Shahkarami, Evolution of Wilson loop in time-dependent $\mathrm{N}=4$ super Yang-Mills plasma. Phys. Rev. D 93(8), 086005 (2016). arXiv:1510.00212 [hep-th]
21. D.S. Ageev, I.Y. Aref'eva, A.A. Golubtsova, E. Gourgoulhon, Holographic Wilson loops in Lifshitz-like backgrounds. arXiv: 1606.03995 [hep-th]

22. A. Hajilou, M. Ali-Akbari, F. Charmchi, A classical string in Lifshitz-Vaidya geometry. Eur. Phys. J. C 78(5), 424 (2018). arXiv:1707.00967 [hep-th]

23. G. Camilo, B. Cuadros-Melgar, E. Abdalla, Holographic thermalization with a chemical potential from Born-Infeld electrodynamics. JHEP 1502, 103 (2015). arXiv:1412.3878 [hep-th]

24. E. Caceres, A. Kundu, Holographic thermalization with chemical potential. JHEP 1209, 055 (2012). arXiv:1205.2354 [hep-th]

25. H. Ebrahim, M. Ali-Akbari, Dynamically probing stronglycoupled field theories with critical point. arXiv:1712.08777 [hepth]

26. R. Critelli, R. Rougemont, J. Noronha, Homogeneous isotropization and equilibration of a strongly coupled plasma with a critical point. JHEP 1712, 029 (2017). arXiv:1709.03131 [hep-th]

27. S.I. Finazzo, J. Noronha, Estimates for the thermal width of heavy quarkonia in strongly coupled plasmas from holography. JHEP 1311, 042 (2013). arXiv:1306.2613 [hep-ph] 